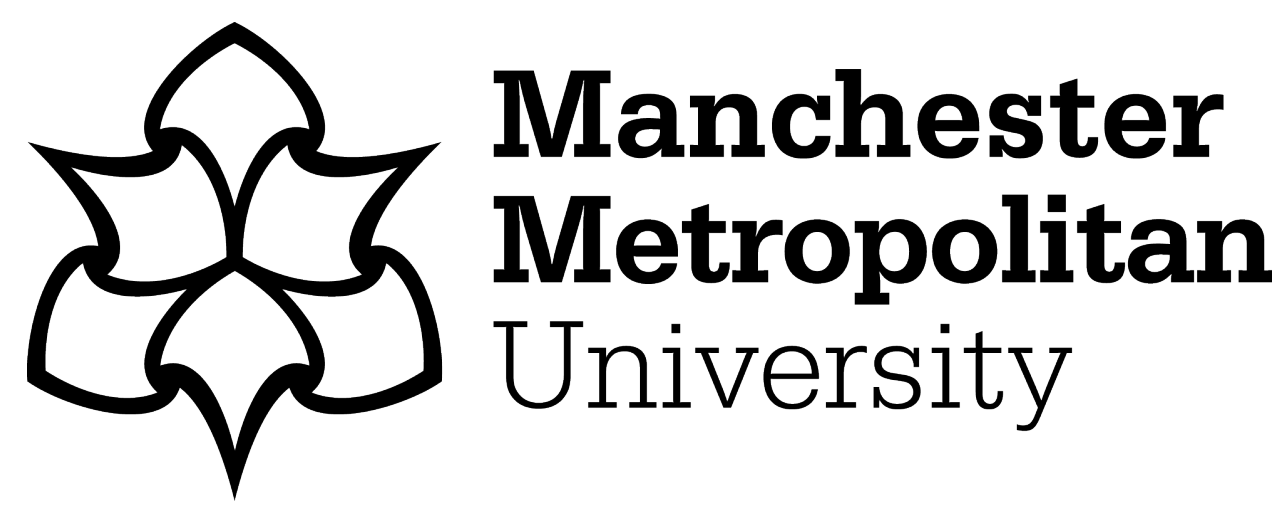

McKeown, M, Chandley, M, Jones, F, Wright, K, Duxbury, J and Foy, P (2016) Explorations of cooperation and recalcitrance in secure mental health hospitals. European Psychiatry, 33 Sup. S462-S462. ISSN 0924-9338

Downloaded from: https://e-space.mmu.ac.uk/621292/

Version: Accepted Version

Publisher: Elsevier

DOI: https://doi.org/10.1016/j.eurpsy.2016.01.1679

Please cite the published version 


\section{Explorations of cooperation and recalcitrance in secure mental health hospitals}

M. McKeown1,*, M. Chandley2, F. Jones1, K. Wright1,

J. Duxbury1, P. Foy2

1 University of Central Lancashire, School of Nursing, Preston, United

Kingdom

2 Mersey Care NHS Trust, Ashworth Hospital, Liverpool, United

Kingdom

* Corresponding author.

This paper reports on the findings of three qualitative research studies undertaken within, respectively, medium and low secure units in one UK region, and a high secure mental health hospital in England (UK). The first study investigated alliance based involvement practices. The second explored service user and staff views and sense making of the notion of recovery. The third study is ongoing into service user and staff experiences of extreme coercive measures, such as forms of restraint and longterm seclusion or segregation. All studies utilised either semi-structured interviews or focus groups. All data was subject to thematic analysis.

Selected and emergent themes include:

- the importance of relationships and communication; security first; involvement as pacification;

- different understandings of recovery; the importance of meaningful occupation; staff-service user relationships; recovery journeys and dialogue with the past; and recovery as personal responsibility;

- good and bad coercion; cooperation, resistance and recalcitrance; and alternatives to coercion.

Findings suggest that cooperation is largely framed by services in terms of compliance with a bio-medical model. The impact of the secure environment, whilst ever-present, is not an absolute constraint on the realisation of recovery or involvement objectives. The availability of extreme coercive measures raises some seemingly paradoxical understandings from both service user and staff perspectives. Critical social theory is drawn on to illuminate the tensions between cooperation and recalcitrance and suggest further exploration of their respective legitimacy.

Disclosure of interest The authors have not supplied their declaration of competing interest. 